\title{
Characteristics and Symptom Severity of Patients Reporting Systemic Lupus Erythematosus in the PatientsLikeMe Online Health Community: A Retrospective Observational Study
}

\author{
Elisabeth Nyman - Timothy Vaughan - Barnabas Desta •
}

Xia Wang · Volkan Barut · Cathy Emmas

Received: November 19, 2019 / Published online: February 1, 2020

(C) The Author(s) 2020

\section{ABSTRACT}

Introduction: Online health communities and research networks such as PatientsLikeMe (PLM) capture patient perspectives of diseases, including systemic lupus erythematosus (SLE). We performed a retrospective observational study of data provided by patients in the PLM SLE community to characterize demographics,

Enhanced Digital Features To view enhanced digital features for this article go to https://doi.org/10.6084/ m9.figshare.11591982.

Electronic Supplementary Material The online version of this article (https://doi.org/10.1007/s40744020-00195-7) contains supplementary material, which is available to authorized users.

E. Nyman

Data Science and AI, AstraZeneca, Göteborg, Sweden

T. Vaughan

Data Sciences, Cohen Veterans Bioscience,

Cambridge, MA, USA

B. Desta

Global Pricing and Market Access, AstraZeneca,

Gaithersburg, MD, USA

X. Wang

Data Science and AI, AstraZeneca, Gaithersburg, MD, USA

V. Barut · C. Emmas ( $₫)$

BioPharmaceuticals Medical, AstraZeneca, Luton, UK

e-mail: cathy.emmas@astrazeneca.com clinical characteristics, patient experience, and symptom impact.

Methods: Adults who registered with PLM in 2011-2017 and reported SLE diagnosis and treatment with one or more SLE-related drug (antimalarials, immunosuppressives, corticosteroids, calcineurin inhibitors, or biologics) were included in the analysis. Information reported within 30 days from PLM registration was used to assess patient eligibility; demographics and clinical characteristics; and primary outcome measures of SLE treatments, symptoms, primary lupus manifestations, and comorbidities.

Results: Among 21,101 PLM members included in this analysis, median ages at registration, onset of SLE symptoms, and SLE diagnosis were 46 years (interquartile range [IQR] 38-53, $n=21,101$ ), 30 years (IQR $21-39 ; n=6489$ ), and 36 years (IQR $27-44 ; n=6936)$, respectively. Most patients were female $(96.8 \%, n=20,370)$. Country of residence was reported by 19,502 patients $(92.4 \%)$, of whom 18,491 (94.8\%) were US residents. Race was recorded by 17,994 patients $(85.3 \%)$, of whom $67.8 \%$ were white and $22.4 \%$ were black/African American. Patients reported a mean of 2.2 SLErelated medications, including antimalarials (83.8\%), corticosteroids (78.8\%), immunosuppressives (32.3\%), and biologics (9.4\%). Fatigue, pain, and joint pain were rated as moderate or severe by at least $80 \%$ of patients who reported these symptoms. Reported primary lupus manifestations and comorbidities included 
fibromyalgia (7.9\%), discoid lupus (6.8\%), lupus nephritis (6.3\%), rheumatoid arthritis (4.8\%), subacute cutaneous lupus (4.7\%), central nervous system lupus (3.9\%), Sjögren's syndrome (3.9\%), and lupus pneumonitis (3.1\%).

Conclusions: Age, sex, and race of patients in the PLM SLE community are broadly consistent with characteristics of the general SLE population in the United States. The PLM SLE population may provide valuable data on selfreported patient experience.

Plain Language Summary: Plain language summary available for this article.

Keywords: Patient perspective; Real-world evidence; Systemic lupus erythematosus; Treatment

\section{Key Summary Points}

\section{Why carry out this study?}

Systemic lupus erythematosus (SLE) presents a substantial disease burden on patients and their quality of life.

Online health communities such as PatientsLikeMe (PLM) capture the patients' perspectives on SLE and provide unique insights into chronic diseases to improve health care.

Characteristics and outcomes of PLM members with SLE were analyzed to evaluate comparability of these patients with the wider SLE population.

\section{What was learned from this study?}

In 21,101 patients comprising the PLM SLE community, demographics, clinical characteristics, SLE medication use, and most frequently reported symptoms (e.g. fatigue and pain) and comorbidities were broadly consistent with the general US SLE population.

The PLM SLE population may be a valuable source of patient-reported data that could be used to obtain insights into patients' experiences and symptoms outside the clinic.

\section{PLAIN LANGUAGE SUMMARY}

Systemic lupus erythematosus (SLE) places a large burden on patients and their quality of life. PatientsLikeMe (PLM) is an online health community that helps patients share information with each other. PLM also collects patients' views on SLE in a way that researchers can use. Research using unique insights from PLM can help to improve the care of patients with SLE. In this study, researchers looked at information from PLM members with SLE to understand their traits (like age, sex, and race) and their disease treatments and symptoms to better understand their experience of SLE. The researchers also wanted to know how patients with SLE who take part in PLM compare with the patients with SLE in other studies. Overall, the traits of the 21,101 patients who were part of the PLM SLE community were similar to those of the general SLE population in the United States. The PLM SLE community also uses similar SLE medications and has similar common symptoms (like fatigue and pain) as the general US SLE population. PLM may be an important source of patient-reported data on SLE. These data could be used to gain new insights into patients' experiences and symptoms.

\section{INTRODUCTION}

Systemic lupus erythematosus (SLE) is an autoimmune disease characterized by chronic inflammation and acute flares, resulting in progressive organ damage and increased mortality $[1,2]$. Reported prevalence of SLE worldwide ranges from 0.3 to 23 per 100,000 person-years [3]. Patients with SLE experience a wide variety of symptoms, including musculoskeletal, mucocutaneous, and neurological symptoms [4]. SLE presents a substantial disease burden, reduced quality of life, and reduced productivity $[5,6]$.

When patients have serious and chronic diseases, they increasingly seek information online and engage in online health communities [7-9]. Online health communities and research networks allow patients to connect with each other, share experiences, and learn 
more about how to manage their condition effectively $[7,10]$. In addition to the value they bring to patients, online health communities and research networks can also provide important insights into chronic diseases to improve health care [11-13].

The online health community PatientsLikeMe (PLM) was designed to help patients interact and share information, as well as capture data in a format that facilitates analysis for research [7]. PLM can be a rich source of patientcentric information. PLM data have the potential to elucidate the patient perspective and can also inform clinical study design. However, to have confidence in the use of these data, it is important to evaluate to what extent this community is representative of the wider SLE population. In this retrospective observational study, we examined data available from PLM members with SLE to characterize demographics, clinical characteristics, symptom severity, primary lupus manifestations, comorbidities, and treatment.

\section{METHODS}

This study was designed to elucidate the experience of patients with SLE. Data utilized in this retrospective observational study were self-reported by patients on the PLM online health community website, a site with more than 600,000 members with more than 2800 conditions [14]. Registration on PLM includes language consenting to the use of patient data for research purposes. PLM's development and research program is guided by a volunteer group of patient advisors from within the PLM community. The results of any research conducted are shared with the PLM community. Independent ethics review was not sought as members of the PLM community are informed of the way their shared data will be used (including for research) via the user agreement and privacy policy before joining the site. Further, as no identifiable information was captured about these anonymous participants, the study falls under Office for Human Research Protections (OHRP) Exempt Categories 45 CFR 46.101(B), i.e. research involving the use of educational tests (cognitive, diagnostic, aptitude, achievement), survey procedures, interview procedures, or observation of public behaviour, and is therefore exempt from ethical approval.

We examined data from patients who registered with PLM between 2011 and 2017. Patients were included in this analysis if they reported a diagnosis of SLE, were aged 18 years or more at registration, and reported treatment with at least one SLE-related medication such as antimalarials, immunosuppressives, corticosteroids, calcineurin inhibitors, or biologics within 30 days of registration. Demographics and clinical characteristics were described for all patients who provided information. We evaluated data on symptom severity, treatment, primary lupus manifestations, and comorbidities reported within 30 days of PLM registration. If multiple reports of the same symptom were entered within 30 days of registration, only the first report was included in these analyses.

Patients who register with PLM are periodically prompted to share a wide variety of health information, including the symptoms they are experiencing under a given diagnosis. Patients who participate on the PLM platform are asked about the severity of their general symptoms, such as anxiety, fatigue, insomnia, stress, and pain. Patients are also asked about symptoms that are specific to their self-identified condition, such as SLE. Over the data collection period, different queries about SLE-related symptoms were added or removed as the website continued to develop. Both general symptoms and SLE-related symptoms were examined in this study. Because patients may choose which data they wish to share, complete data are not available for all patients. No attempt was made to correct for missing data.

The prednisone-equivalent daily dose was calculated for oral corticosteroid treatments [15] when information on dose and frequency was available and if the reported start date was within 30 days from PLM registration. If multiple treatment reports were identified with a reported start date within 30 days of registration, the highest treatment dose was used. Reported prednisolone-equivalent dosages 
greater than $300 \mathrm{mg} /$ day were excluded from the analysis as being unrealistically high.

Detailed disease and clinical characteristics of patients with SLE registered with PLM are reported using summary statistics. Continuous variables are presented as medians and interquartile ranges or means and standard deviations (SDs). Categorical variables are summarized as counts and percentages. No tests of statistical significance were performed.

\section{RESULTS}

\section{Patient Demographics}

More than 600,000 patients were registered with PLM during 2011-2017 when this study was conducted, including more than 29,000 with self-reported SLE. Of these, 21,101 adults met criteria to be included in this analysis (Fig. 1). For the patients in this analysis, median age at registration was 46 years, median age at onset of SLE symptoms was 30 years, and median age at diagnosis was 36 years (Table 1 ). Mean time between reported symptom onset and diagnosis was 5.4 years $(n=6245)$. Most
(96.8\%) PLM members in this analysis were women. Patients who reported a country of residence lived in 75 different countries. Most patients $(94.8 \%)$ resided in the United States; the next most commonly reported countries were the UK $(2.3 \%)$, Canada (1.0\%), Australia (0.4\%), and South Africa and Puerto Rico (0.2\% each), as well as Ireland, New Zealand, India, Bahamas, and Indonesia $(0.1 \%$ each). Most patients who reported race were white $(67.8 \%)$; $22.4 \%$ were black or African American. Of the 17,371 patients who reported ethnicity, $13.3 \%$ were Hispanic or Latino.

\section{SLE Medications}

SLE medications were recorded by all patients $(N=21,101)$ within 30 days of PLM registration, with a mean of 2.2 medications (SD 1.1) reported per patient. Antimalarial medication was the most frequently reported medication (83.8\%) (Fig. 2), and use of corticosteroids was reported by $78.8 \%$ of patients; $78.0 \%$ reported using prednisone (Table S1 in the supplementary material). Use of immunosuppressive medications was reported by $32.3 \%$ of patients

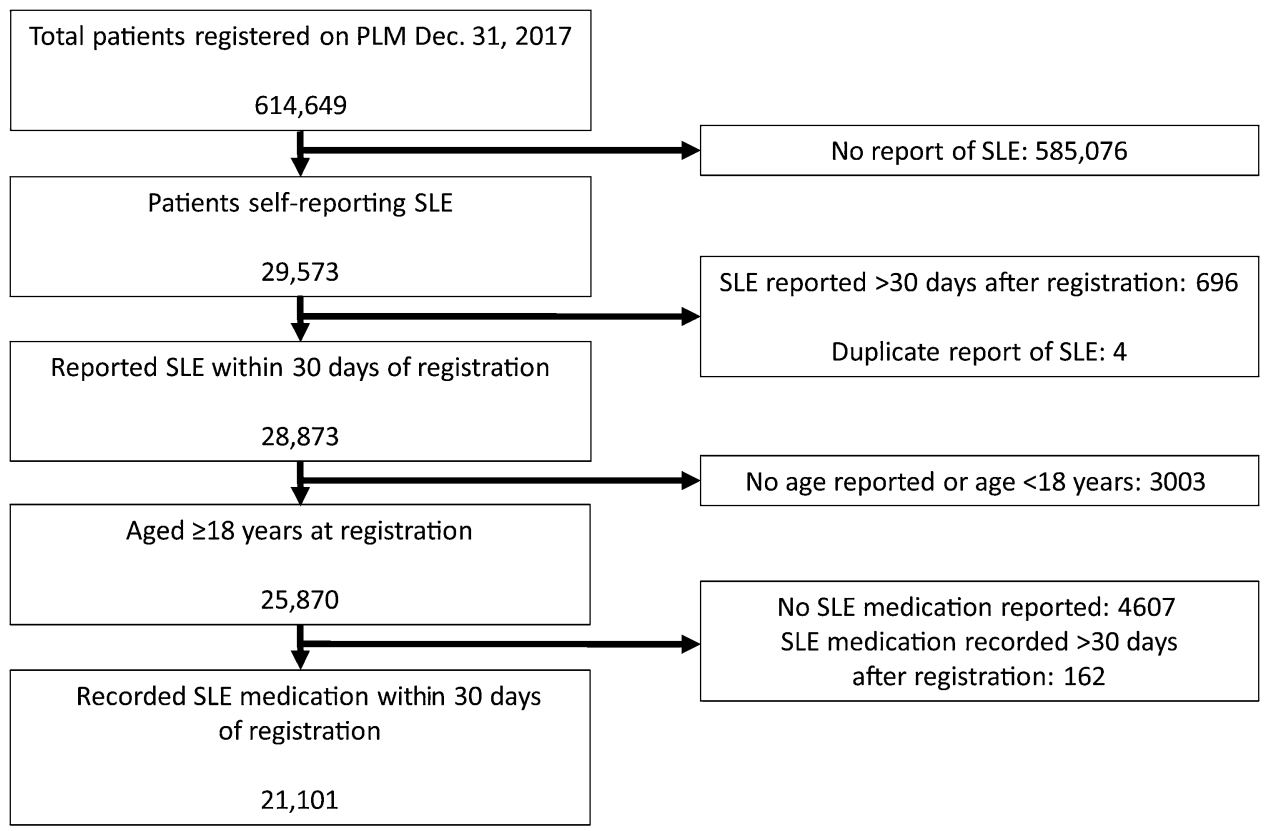

Fig. 1 Patient selection from the PatientsLikeMe community. PLM PatientsLikeMe, SLE systemic lupus erythematosus 
Table 1 Characteristics of PLM members with SLE (2011-2017) included in analyses

\begin{tabular}{|c|c|c|}
\hline Characteristics & Median (IQR) & $N$ \\
\hline Age at PLM registration [years] & $46(38-53)$ & 21,101 \\
\hline Age at first SLE symptoms [years] & $30(21-39)$ & 6489 \\
\hline \multirow[t]{2}{*}{ Age at SLE diagnosis [years] } & $36(27-44)$ & 6936 \\
\hline & $n(\%)$ & $N$ \\
\hline Women & $20,370(96.8)$ & 21,050 \\
\hline Country of residence & & 19,502 \\
\hline United States & $18,491(94.8)$ & \\
\hline UK & $442(2.3)$ & \\
\hline Canada & $203(1.0)$ & \\
\hline Other & $366(1.9)$ & \\
\hline Race & & 17,994 \\
\hline White & $12,191(67.8)$ & \\
\hline Black or African American & $4026(22.4)$ & \\
\hline Mixed race & $1139(6.3)$ & \\
\hline American Indian or Alaskan Native & $333(1.9)$ & \\
\hline Asian & $235(1.3)$ & \\
\hline Native Hawaiian or other Pacific Islander & $70(<1.0)$ & \\
\hline Hispanic or Latino ethnicity & $2308(13.3)$ & 17,371 \\
\hline Education & & 6400 \\
\hline High school or less & $1145(17.9)$ & \\
\hline Some college & $3065(47.9)$ & \\
\hline Undergraduate or higher degree & $2190(34.2)$ & \\
\hline Health insurance & & 6029 \\
\hline Commercial & $2850(47.3)$ & \\
\hline Medicare & $1338(22.2)$ & \\
\hline Medicaid & $945(15.7)$ & \\
\hline Uninsured & $321(5.3)$ & \\
\hline Other & $575(9.5)$ & \\
\hline
\end{tabular}

IQR interquartile range, PLM PatientsLikeMe, SLE systemic lupus erythematosus

(use of methotrexate, mycophenolate mofetil, and azathioprine was reported in 19.8\%, 9.6\%, and $9.1 \%$ of patients, respectively). Use of biologic medications was reported by $9.4 \%$ of patients; $8.4 \%$ of patients reported using belimumab. 


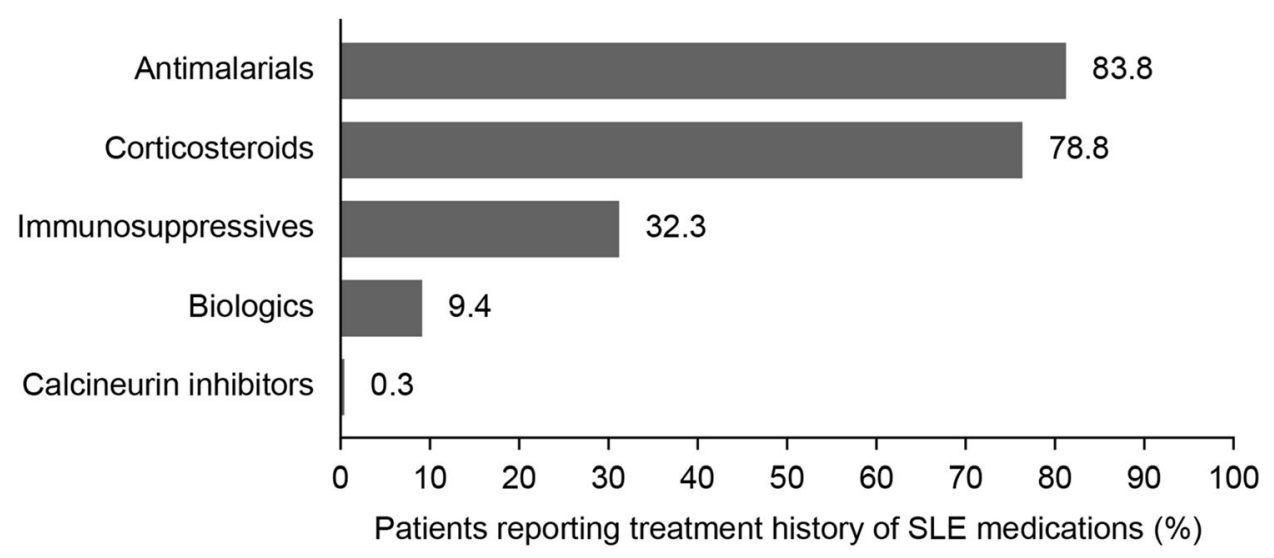

Fig. 2 Percentage of patients with SLE reporting each medication within 30 days of PLM registration. PLM PatientsLikeMe, SLE systemic lupus erythematosus

Of the 16,632 patients who indicated use of corticosteroids, 909 (5.5\%) included sufficient information to allow total daily steroid dosage to be calculated. Median reported prednisoneequivalent dosage for these 909 patients was $20 \mathrm{mg} /$ day (IQR 10-50 mg/day); 167 (18.4\%) received at most $7.5 \mathrm{mg} /$ day, 417 (45.9\%) received more than 7.5 to $30 \mathrm{mg} /$ day, 299 (32.9\%) received more than 30 to $100 \mathrm{mg} /$ day, and $26(2.9 \%)$ received more than $100 \mathrm{mg} /$ day.

\section{Symptoms}

Thirty-one percent of patients $(n=6444)$ reported either general or SLE-related symptom information within 30 days of PLM registration. The intensity of each general symptom, ranging from none to severe, was rated by $6122-6387$ patients. The three general symptoms for which moderate or severe symptom severity was most commonly reported were fatigue $(84.9 \%)$, pain (80.0\%), and insomnia (61.1\%; Fig. 3). Each SLE-related symptom was reported by 407-6138 patients. The SLE-related symptoms for which moderate or severe symptom severity was most commonly reported were joint pain $(81.5 \%)$, muscle pain $(71.8 \%)$, and muscle weakness (60.1\%; Fig. 3). In contrast, Raynaud's phenomenon, butterfly (malar) rash, and chest pain were the symptoms that patients most frequently indicated as being absent (47.7\%, $44.6 \%$, and $42.6 \%$, respectively, of patients who provided information on those symptoms reported that they were absent).

\section{Comorbidities and Primary Lupus Manifestations}

Among the 21,101 patients with SLE on PLM, the comorbidities reported included fibromyalgia (7.9\%), rheumatoid arthritis (4.8\%), Sjögren's syndrome $(3.9 \%)$, and generalized anxiety disorder (2.3\%; Fig. 4). Discoid lupus (6.8\%), lupus nephritis $(6.3 \%)$, and subacute cutaneous lupus $(4.7 \%)$ were the most commonly reported primary lupus manifestations (Fig. 4).

\section{DISCUSSION}

Overall, analysis of a large dataset of 21,101 PLM members with SLE revealed valuable insight into the patient experience and the impact of SLE symptoms. The characteristics of the PLM members with SLE who were included in this analysis were similar to those of the general SLE population in the United States. Almost 97\% of patients with SLE in the PLM community between 2011 and 2017 were women. This is somewhat higher than the range of $82-93 \%$ reported in real-world samples of patients with SLE [1, 16-22]. This is consistent, however, with the report that members of another large online community of patients with SLE were also 97\% female [23] and that 


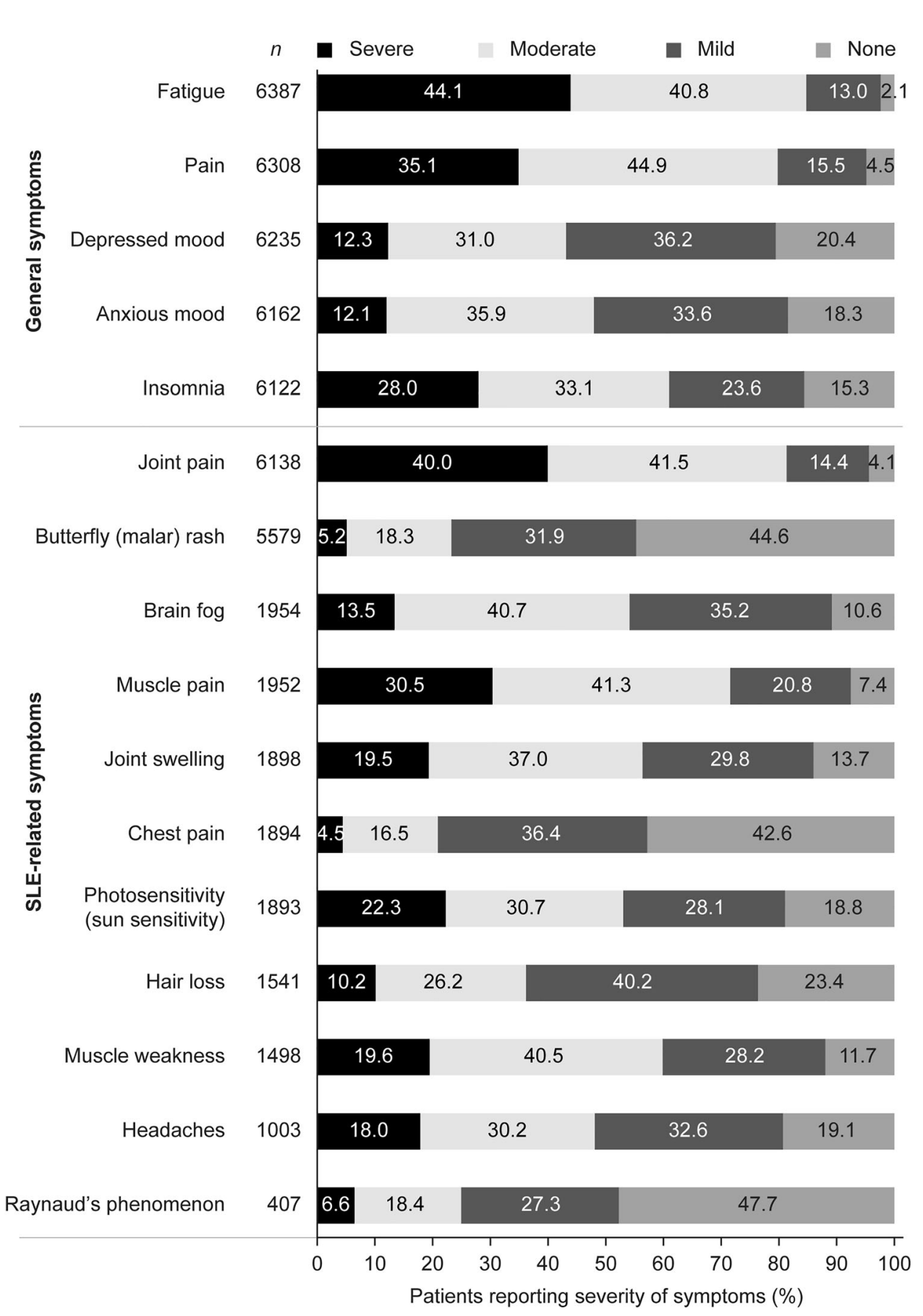

Fig. 3 Severity of symptoms reported by patients with SLE within 30 days of PLM registration. For each symptom listed in the panel, patients had the option to select severe, moderate, mild, or none. $N$ values indicate the number of patients who selected any answer for each

women are more likely than men to seek health information from the Internet [24-26]. Approximately $95 \%$ of the patients with SLE individual symptom. The percentage of responding patients who chose each option is presented in the bars to the right. PLM PatientsLikeMe, SLE systemic lupus erythematosus

who reported a country of residence live in the United States. This is not surprising given that PLM is based in the United States and is an 


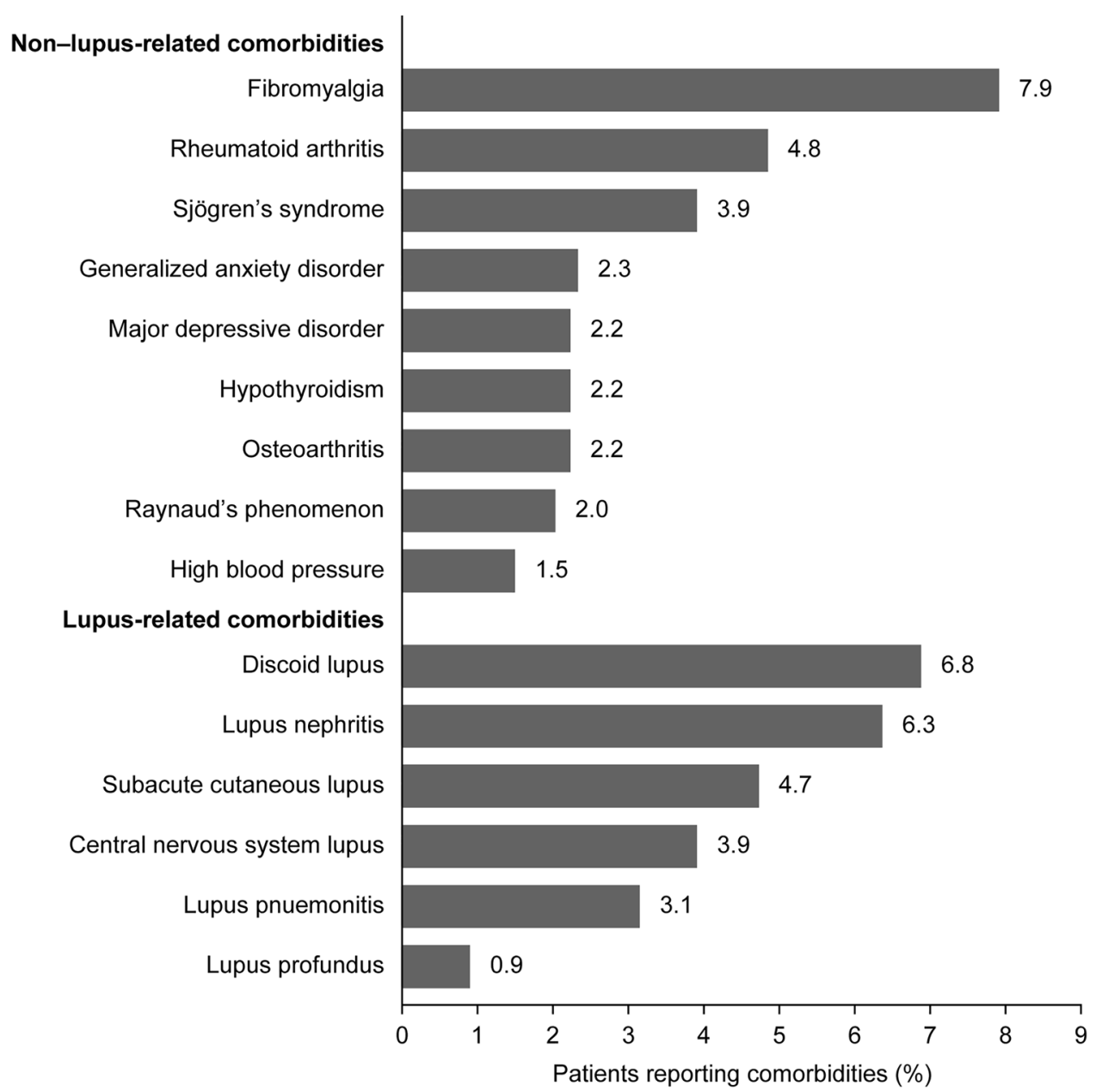

Fig. 4 Comorbidities and primary lupus manifestations reported by patients with SLE within 30 days of registration on PLM. Figure shows the percentage of the 21,101

English-language website. However, there is some worldwide reach for PLM as reflected in the 75 countries that are represented by at least one patient each in the PLM SLE community.

Race among patients with SLE varies with the country or geographic area studied; SLE prevalence studies in three different regions of the United States and in the US Medicare and Medicaid populations reported ranges of $20-77 \%$ for black or African American patients and $22-63 \%$ for white patients $[16,17,19-22]$. Among patients in the PLM SLE community who reported race, the percentage of black or African American patients is at the low end of this range $(22 \%)$, whereas white patients are somewhat over-represented (68\%). However, patients in this analysis who reported each comorbidity. PLM PatientsLikeMe, SLE systemic lupus erythematosus

white adults are known to be more likely to search for health information online than adults of other races [24]. Hispanic ethnicity among patients with SLE is also widely variable in published reports (range 2-32\%) $[16,19,21,22]$; the percentage of PLM members with SLE reporting Hispanic ethnicity falls in the middle of this range (13\%). The socioeconomic status of the PLM members was comparable with that reported in other non-online SLE populations. The percentage of PLM patients with SLE with at least a college undergraduate/bachelor degree was $34.2 \%$, which is consistent with the range of $38-39 \%$ reported in other SLE cohorts $[27,28]$. Similarly, the proportion of patients enrolled in Medicaid and/or 
Medicare (or equivalent) was $38 \%$ in our study, which is within the range of $32-43 \%$ reported in other SLE cohorts [27, 28].

Age at symptom onset and age at diagnosis of SLE are comparable to ranges previously reported, and the delay between symptom onset and duration is consistent with previous findings $[4,20,21,29]$. The types of symptoms reported by PLM members are also consistent with those observed in the overall lupus population [16, 19-21, 30]. Many lupus studies do not collect information on fatigue. However, recent studies have shown that patients with SLE report fatigue and pain as symptoms with the highest impact [23, 31]. A study of 569 questionnaire results from another online community of patients with SLE reported that fatigue was the most frequently reported symptom of great impact, followed by joint pain and other pain [23]. This is consistent with the reports of patients in the PLM SLE community, who reported high levels of severe fatigue $(44.1 \%)$, pain $(35.1 \%)$, and joint pain (40.0\%). Among patients who reported symptoms, many reported that they were not experiencing the SLE-related symptoms of Raynaud's phenomenon $(47.7 \%)$, butterfly rash $(44.6 \%)$, or chest pain $(42.6 \%)$. It should be noted that the specific data collected during the history of the PLM website (and therefore the study period) has changed. General symptoms have been collected since the inception of the website, whereas different condition-specific symptoms have been added or removed over time. This likely impacted reporting rates for these symptoms, resulting in over-representation of the type 2 non-inflammatory lupus symptoms (including general symptoms such as fatigue, widespread pain, and cognitive dysfunction) compared with the type 1 inflammatory symptoms (including SLE-specific symptoms such as nephritis, inflammatory arthritis, and cutaneous rash) [32].

Medication patterns within the PLM SLE community are similar to those reported in studies of patients with SLE, with antimalarials and corticosteroids being most commonly prescribed $[33,34]$. The median reported corticosteroid dosage for PLM members with SLE was $20 \mathrm{mg} /$ day. Other studies have reported mean daily doses of 9-24 mg [1, 34]. Although the median prednisone dosage was consistent with previous reports $[1,34]$, we noted a relatively high percentage $(36 \%)$ of patients who indicated use of prednisone greater than $30 \mathrm{mg} /$ day among the subset of patients who reported their prednisone dosage. Although this dosage is higher than expected, patients may be more likely to report prednisone dosage if a high dosage represented a significant burden for them.

The low number of comorbidities listed for patients in the PLM SLE community suggests that most patients who experienced comorbidities and primary lupus manifestations chose not to report them. Fibromyalgia, arthritis, and Sjögren's syndrome, the most common of the non-lupus-related comorbidities reported by PLM members with SLE, are also common in the overall lupus population [16, 19-21, 30].

Patients registered on the PLM platform have awareness of and consent to the use of their data for research. This may influence what information patients choose to share on the PLM platform. The recorded symptoms and diagnosis were reliant on patient self-report, and lupus diagnoses were not confirmed in this analysis. Patients were neither required nor expected to complete all data fields available; therefore, some data may have been missed or misrepresented. However, PLM allows collection of data from people who might not agree to participate in a more regimented system, such as a patient registry. Although data derived from patient self-report may benefit from external validation, in a study of consenting patients registered on PLM, there was a 93\% agreement rate between patient-reported multiple sclerosis diagnosis and information on electronic medical records [35].

A further limitation is that some patient subpopulations appear to be under-represented in the PLM SLE community compared with the general SLE population in the United States. This may be due to an over-representation of the demographic most likely to use online communities (e.g. white, female, of a younger age, with college or advanced degree) [24]. Under-representation of minority patients, including black/African American patients, 
patients with very severe disease, and patients with limited computer access, may result in an underestimate of symptom severity. However, socio-economic factors (education, health insurance) were comparable to other non-online US populations, suggesting that the PLM community may not be biased toward certain patient demographics [27, 28]. Although no formal statistical analyses of subgroups by race were performed, there were some indications of differing responses between black/African American and white patients, which merit further investigation. Although PLM is not designed with the purpose of achieving a patient sample that is representative of any particular real-world disease population, data from this online health community can nonetheless generate insights into patients' experiences regarding their conditions.

Whilst there are some limitations to the data, PLM does provide a unique source of realworld information on patient experience with symptoms of SLE outside the clinical environment and may provide more focus on those symptoms that are especially impactful for patients. Multiple studies have identified direct benefits for patients using PLM, including improved disease self-management capabilities and higher levels of quality of life [36-38]. Unlike data from electronic health records, PLM data are not filtered through a physician's perception and judgement. Although this means there is no official diagnosis captured and medication dosage information may be lacking, it may also mean better information about symptom severity and access to unedited patient perspectives.

\section{CONCLUSIONS}

PLM is designed primarily to help patients connect with and learn from each other. However, the data and experience patients shared in this online community can also be used to improve understanding of SLE and support the future design of clinical research and patient services to best meet the needs of patients. The large size of the PLM SLE population and its general similarity to the wider SLE population provide opportunities for future research to improve understanding of patient experience and perspective.

\section{ACKNOWLEDGEMENTS}

The authors thank the patients in the PLM SLE community for their willingness to share their experiences and help advance research.

Funding. This work and the Rapid Service Fee were supported by AstraZeneca.

Medical Writing and Editorial Assistance. Writing and editing assistance was provided by Jennie G. Jacobson, PhD, CMPP, of JK Associates, Inc., a member of the Fishawack Group of Companies, Conshohocken, PA, USA. This support was funded by AstraZeneca.

Authorship. All named authors meet the International Committee of Medical Journal Editors (ICMJE) criteria for authorship for this article, take responsibility for the integrity of the work as a whole, and have given their approval for this version to be published.

Authorship Contributions. VB, BD, CE, EN, and $\mathrm{XW}$ contributed to conception and design of the study. TV contributed to collection of data. All authors contributed to data analysis and interpretation and to manuscript preparation and review. All authors approved the final version of the manuscript, and agree to be accountable for all aspects of the work.

Disclosures. Timothy Vaughan was an employee of PatientsLikeMe at the time of the study and is now an employee of Data Sciences, Cohen Veterans Bioscience, Cambridge, MA, USA. Elisabeth Nyman, Barnabas Desta, Xia Wang, Volkan Barut, and Cathy Emmas are employees of AstraZeneca.

Compliance with Ethics Guidelines. Independent ethics review was not sought as members of the PatientsLikeMe community are informed of the way their shared data will be used (including for research) via the user 
agreement and privacy policy before joining the site. Further, as no identifiable information was captured about these anonymous participants, the study falls under Office for Human Research Protections (OHRP) Exempt Categories 45 CFR 46.101(B), i.e. research involving the use of educational tests (cognitive, diagnostic, aptitude, achievement), survey procedures, interview procedures, or observation of public behavior, and is therefore exempt from ethical approval.

Data Availability. The data that support the findings of this study were collected from PatientsLikeMe. Restrictions apply to the availability of these data, which were used under license for this study.

Open Access. This article is distributed under the terms of the Creative Commons Attribution-NonCommercial 4.0 International License (http://creativecommons.org/licenses/ by-nc/4.0/), which permits any noncommercial use, distribution, and reproduction in any medium, provided you give appropriate credit to the original author(s) and the source, provide a link to the Creative Commons license, and indicate if changes were made.

\section{REFERENCES}

1. Bruce IN, $\mathrm{O}^{\prime}$ Keeffe AG, Farewell V, et al. Factors associated with damage accrual in patients with systemic lupus erythematosus: results from the Systemic Lupus International Collaborating Clinics (SLICC) Inception Cohort. Ann Rheum Dis. 2015;74:1706-13. https://doi.org/10.1136/annrhe umdis-2013-205171.

2. Jorge AM, Lu N, Zhang Y, et al. Unchanging premature mortality trends in systemic lupus erythematosus: a general population-based study (1999-2014). Rheumatology (Oxford). 2018;57: 337-44. https://doi.org/10.1093/rheumatology/ kex412.

3. Rees F, Doherty M, Grainge MJ, et al. The worldwide incidence and prevalence of systemic lupus erythematosus: a systematic review of epidemiological studies. Rheumatology (Oxford). 2017;56:1945-61. https://doi.org/10.1093/rheumatology/kex260.
4. Nightingale AL, Davidson JE, Molta CT, et al. Presentation of SLE in UK primary care using the Clinical Practice Research Datalink. Lupus Sci Med. 2017;4:e000172. https://doi.org/10.1136/lupus2016-000172.

5. Meacock R, Dale N, Harrison M. The humanistic and economic burden on systemic lupus erythematosus. Pharmacoeconomics. 2013;31:49-61. https://doi.org/10.1007/s40273-012-0007-4.

6. Utset TO, Baskaran A, Segal BM, et al. Work disability, lost productivity and associated risk factors in patients diagnosed with systemic lupus erythematosus. Lupus Sci Med. 2015;2:e000058. https:// doi.org/10.1136/lupus-2014-000058.

7. Wicks P, Hixson J. The patient engagement pill: lessons from epilepsy. 2013. https://www. healthaffairs.org/do/10.1377/hblog20130207. 027850/full. Accessed May 31, 2019.

8. Tan SSL, Goonawardene N. Internet health information seeking and the patient-physician relationship: a systematic review. J Med Internet Res. 2017;19:e9. https://doi.org/10.2196/jmir.5729.

9. An LC, Wallner L, Kirch MA. Online social engagement by cancer patients: a clinic-based patient survey. JMIR Cancer. 2016;2:e10. https:// doi.org/10.2196/cancer.5785.

10. Wicks P, Mack Thorley E, Simacek K, et al. Scaling PatientsLikeMe via a "generalized platform" for members with chronic illness: web-based survey study of benefits arising. J Med Internet Res. 2018;20:e175. https://doi.org/10.2196/jmir.9909.

11. Little M, Wicks P, Vaughan T, et al. Quantifying short-term dynamics of Parkinson's disease using self-reported symptom data from an Internet social network. J Med Internet Res. 2013;15:e20. https:// doi.org/10.2196/jmir.2112.

12. Wicks P, Vaughan TE, Massagli MP, et al. Accelerated clinical discovery using self-reported patient data collected online and a patient-matching algorithm. Nat Biotechnol. 2011;29:411-4. https://doi. org/10.1038/nbt.1837.

13. Eaneff S, Wang V, Hanger $M$, et al. Patient perspectives on neuromyelitis optica spectrum disorders: data from the PatientsLikeMe online community. Mult Scler Relat Disord. 2017;17: 116-22. https://doi.org/10.1016/j.msard.2017.07. 014.

14. PatientsLikeMe. https://www.patientslikeme.com. Accessed Mar 22, 2019.

15. Parente L. Deflazacort: therapeutic index, relative potency and equivalent doses versus other 
corticosteroids. BMC Pharmacol Toxicol. 2017;18: 1. https://doi.org/10.1186/s40360-016-0111-8.

16. Dall'Era M, Cisternas MG, Snipes K, et al. The incidence and prevalence of systemic lupus erythematosus in San Francisco county, California: the California Lupus Surveillance Project. Arthritis Rheumatol. 2017;69:1996-2005. https://doi.org/10. 1002/art.40191.

17. Garris C, Shah M, Farrelly E. The prevalence and burden of systemic lupus erythematosus in a Medicare population: retrospective analysis of Medicare claims. Cost Eff Resour Alloc. 2015;13:9. https://doi.org/10.1186/s12962-015-0034-z.

18. Ribi C, Trendelenburg M, Gayet-Ageron A, et al. The Swiss systemic lupus erythematosus cohort study (SSCS)—cross-sectional analysis of clinical characteristics and treatments across different medical disciplines in Switzerland. Swiss Med Wkly. 2014;144:w13990. https://doi.org/10.4414/smw. 2014.13990 .

19. Izmirly PM, Wan I, Sahl S, et al. The incidence and prevalence of systemic lupus erythematosus in New York county (Manhattan), New York: the Manhattan Lupus Surveillance Program. Arthritis Rheumatol. 2017;69:2006-17. https://doi.org/10.1002/art. 40192 .

20. Lim SS, Bayakly AR, Helmick CG, et al. The incidence and prevalence of systemic lupus erythematosus, 2002-2004: the Georgia Lupus Registry. Arthritis Rheumatol. 2014;66:357-68. https://doi. org/10.1002/art.38239.

21. Somers EC, Marder W, Cagnoli P, et al. Populationbased incidence and prevalence of systemic lupus erythematosus: the Michigan Lupus Epidemiology and Surveillance program. Arthritis Rheumatol. 2014;66:369-78. https://doi.org/10.1002/art.38238.

22. Li T, Carls GS, Panopalis P, et al. Long-term medical costs and resource utilization in systemic lupus erythematosus and lupus nephritis: a five-year analysis of a large Medicaid population. Arthritis Rheum. 2009;61(6):755-63. https://doi.org/10. 1002/art.24545.

23. Hall SA, Chen SY, Kao A, et al. Symptom impact and unmet need in systemic/cutaneous lupus erythematosus: results from a patient-centred study set in a social media community. Lupus Sci Med. 2017;4(Suppl 1):A77. https://doi.org/10.1136/lupus2017-000215.162.

24. Fox S, Duggan M. Health Online 2013. Pew Research Center's Internet and American Life Project. 2013. https://www.pewinternet.org/2013/01/ 15/health-online-2013. Accessed May 31, 2019.
25. Bidmon S, Terlutter R. Gender differences in searching for health information on the internet and the virtual patient-physician relationship in Germany: exploratory results on how men and women differ and why. J Med Internet Res. 2015;17: e156. https://doi.org/10.2196/jmir.4127.

26. Montagni I, Cariou T, Feuillet T, et al. Exploring digital health use and opinions of university students: field survey study. JMIR mHealth uHealth. 2018;6:e65. https://doi.org/10.2196/mhealth.9131.

27. Tonner C, Trupin L, Yazdany J, et al. Role of community and individual characteristics in physician visits for persons with systemic lupus erythematosus. Arthritis Care Res (Hoboken). 2010;62:888-95. https://doi.org/10.1002/acr.20125.

28. Yelin E, Trupin L, Katz P, et al. Impact of health maintenance organizations and fee-for-service on health care utilization among people with systemic lupus erythematosus. Arthritis Rheum. 2007;57: 508-15. https://doi.org/10.1002/art.22625.

29. Murphy G, Isenberg D. Effect of gender on clinical presentation in systemic lupus erythematosus. Rheumatology (Oxford). 2013;52:2108-15. https:// doi.org/10.1093/rheumatology/ket160.

30. Torrente-Segarra V, Salman-Monte TC, Rua-Figueroa I, et al. Fibromyalgia prevalence and related factors in a large registry of patients with systemic lupus erythematosus. Clin Exp Rheumatol. 2016;34(2 Suppl 96): S40-7.

31. Eaneff S, Vaughan T, Barut V, et al. How do patients describe their "new normal" in systematic lupus erythematosus? Use of probabilistic topic modeling to characterize patients' experiences recorded in an online health community [abstract]. Arthritis Rheum. 2018;70(Suppl 10).

32. Pisetsky DS, Clowse MEB, Criscione-Schreiber LG, et al. A novel system to categorize the symptoms of systemic lupus erythematosus. Arthritis Care Res. 2019;71(6):735-41. https://doi.org/10.1002/acr.237 94.

33. Kan H, Nagar S, Patel J, et al. Longitudinal treatment patterns and associated outcomes in patients with newly diagnosed systemic lupus erythematosus. Clin Ther. 2016;38:610-24. https://doi.org/10. 1016/j.clinthera.2016.01.016.

34. Hanly JG, Sayani A, Doucette S, et al. Treatment pathways in an inception lupus cohort over the first three years. Lupus. 2017;26:119-24. https://doi.org/ $10.1177 / 0961203316655213$.

35. Eichler GS, Cochin E, Han J, et al. Exploring concordance of patient-reported information on PatientsLikeMe and medical claims data on the 
patient level. J Med Internet Res. 2016;18:e110. https://doi.org/10.2196/jmir.5130.

36. Frost JH, Massagli MP. Social uses of personal health information within PatientsLikeMe, an online patient community: what can happen when patients have access to one another's data. J Med Internet Res. 2008;10(3):e15. https://doi.org/10. 2196/jmir.1053.
37. Wicks P, Massagli M, Frost J, et al. Sharing health data for better outcomes on PatientsLikeMe. J Med Internet Res. 2010;12(2):e19. https://doi.org/10. 2196/jmir.1549.

38. Hixson JD, Barnes D, Parko K, et al. Patients optimizing epilepsy management via an online community: the POEM Study. Neurology. 2015;85(2):129-36. https://doi.org/10.1212/WNL.0000000000001728. 